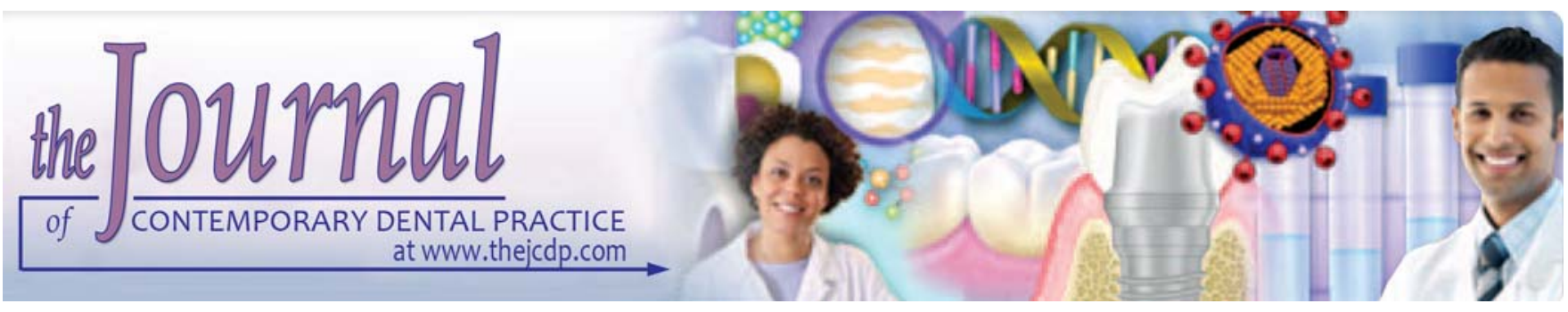

\title{
Grape Seed Extract as a Potential Remineralizing Agent: A Comparative in vitro Study
}

\author{
Shiny Benjamin, Roshni, Sabeena Susan Thomas, Mohan Thomas Nainan
}

\begin{abstract}
Objective: Remineralization is an effective treatment that may stop or reverse early tooth decay. Grape seed extract (GSE) is the potential remineralizing agent under investigation.

Materials and methods: Sound human tooth sections were obtained from the cervical portion of the root and stored in demineralizing solution at $37^{\circ} \mathrm{C}$ for 96 hours to induce artificial root caries lesions. The sections were divided into four treatment groups including $6.5 \%$ grape seed extract, sodium monofluorophosphate $(220 \mathrm{ppm})$ with $0.05 \%$ calcium glycerophosphate, $0.5 \%$ calcium glycerophosphate and control (no treatment). An in vitro $\mathrm{pH}$ cycling model was used to cycle the demineralized specimens through treatment solutions, acidic buffer and neutral buffer for 8 days at 6 cycles per day. Subsequently, they were evaluated using confocal laser scanning microscope. Data were analyzed using analysis of variance $(p<0.05)$.
\end{abstract}

Results: GSE revealed less demineralization and more remineralization compared with other groups.

Conclusion: GSE promotes remineralization of artificial root caries lesions.

Clinical significance: The search for the perfect remineralizing agent continues to this day. GSE could be a welcome addition to the remineralization armamentarium.

Abbreviations and acronyms: GSE: Grape seed extract; ppm: Parts per million; CaGP: Calcium glycerophosphate; CLSM: Confocal laser scanning microscope; ANOVA: Analysis of variance; $\mathrm{PA}$ : P roanthocyanidin; $\mathrm{CEJ}$ : Cementoenamel junction; $\mathrm{mM}$ : Millimole; $\mathrm{CaCl}_{2} 2 \mathrm{H}_{2} \mathrm{O}$ : Calcium chloride dihydrate; $\mathrm{KH}_{2} \mathrm{PO}_{4}$ : Potassium dehydrate phosphate; $\mathrm{K}_{2} \mathrm{HPO}_{4}$ : Dipotassium phosphate; $\mathrm{dH}_{2} \mathrm{O}$ : Deionized water; w/v: Weight by volume; ROD: R elative optical density; nm: Nanometer; SD: Standard deviation.

Keywords: R emineralization, Root caries, Grape seed extract, Confocal laser scanning microscope, Proanthocyanidin, In vitro $\mathrm{pH}$ cycling model, Laboratory research.

How to cite this article: Benjamin S, Roshni, Thomas SS, Nainan MT. Grape Seed Extract as a Potential Remineralizing Agent: A Comparative in vitro Study. J Contemp Dent Pract 2012;13(4):425-430.

Source of support: Nil

Conflict of interest: None declared

\section{INTRODUCTION}

Prevention of extension by remineralization of caries is highly desirable and is one of the cornerstones of minimal invasive dentistry. ${ }^{1}$ Caries starts as demineralization followed by destruction of organic matter and invasion by microorganisms. ${ }^{2-4}$ Remineralization is the repair of established carious lesions. Structurally intact collagen may provide a good scaffold to promote remineralization. A n agent which strengthens collagen-based tissues by increasing collagen cross-links would promote remineralization. ${ }^{5}$

Polyphenols are plant-derived substances which have antioxidant and anti-inflammatory properties. ${ }^{6-8}$ Polyphenols are able to interact with microbial membrane proteins, enzymes and lipids thereby altering cell permeability and permitting the loss of proteins, ions and macromolecules. One such polyphenol is proanthocyanidin (PA) which is a bioflavanoid-containing benzene-pyran-phenolic acid molecular nucleus. ${ }^{7}$

Proanthocyanidin, a natural ly occurring plant metabolite is a natural antioxidant and free radical scavenger. The PA accelerates the conversion of soluble collagen to insoluble collagen during development and increases collagen synthesis. $^{6}$

The present study, aimed to analyze the remineralization potential of grape seed extract (GSE) which contains PA on root carious lesions using an in vitro pH cycling model.

\section{MATERIALS AND METHODS}

\section{Specimen Preparation}

Twenty sound human teeth extracted for periodontal reasons were obtained. They were cleaned and the organic contaminants were removed with an ultrasonic scaler (Piezoelectric ultrasonic scaler ART - P1, B onart Co Ltd, Taiwan). Forty root fragments from below the CEJ measuring about $4 \times 4 \mathrm{~mm}$ were obtained and polished with 
a polishing disk (Sof-lex, 3M ESPE) and used in the study. Root fragments were sealed with an acid resistant nail polish (Revlon) except for a $3 \times 2 \mathrm{~mm}$ window (Figs 1 and 2; measurements done using an acrylic template).

\section{Lesion Formation}

Root fragments were placed in a demineralizing solution [2.2 mM CaCl $2.2 \mathrm{H}_{2} \mathrm{O}$ (T- Baker Lab Chemicals, India), $2.2 \mathrm{mM} \mathrm{KH}_{2} \mathrm{PO}_{4}$ (S D Fine Chem Ltd, M umbai, India), $50 \mathrm{mM}$ A cetate (SD Fine Chem Ltd, M umbai, India), $\mathrm{pH}$ 4.6] for 96 hours at $37^{\circ} \mathrm{C}$ to create artificial carious lesions. Following lesion development, the fragments were rinsed thoroughly with deionized water $\left(\mathrm{dH}_{2} \mathrm{O}\right)$.

\section{Remineralization Tegimen}

The demineralized root fragments were randomly divided into four groups $(n=10)$ based on treatments as follows:

- Group A: 6.5\% (w/v) GSE solution prepared in phosphate buffer $\left[0.025 \mathrm{M} \mathrm{KH}_{2} \mathrm{PO}_{4}, 0.025 \mathrm{M} \mathrm{K}_{2} \mathrm{HPO}_{4}\right.$ (S D Fine Chem Ltd, M umbai, India) at pH 7.4]. GSE purchased from Zenith Nutrition, M edizen Labs, India.

- Group B: $0.05 \%$ CaG P (Chemsw orth, Surat, India) with $0.17 \%$ sodium monofluorophosphate (NR Chem, M umbai, India).

- Group C: $0.5 \%$ CaGP

- Group D: Deionized water ( $\left.\mathrm{dH}_{2} \mathrm{O}\right)$, (ELLIX, M illipore) no treatment.

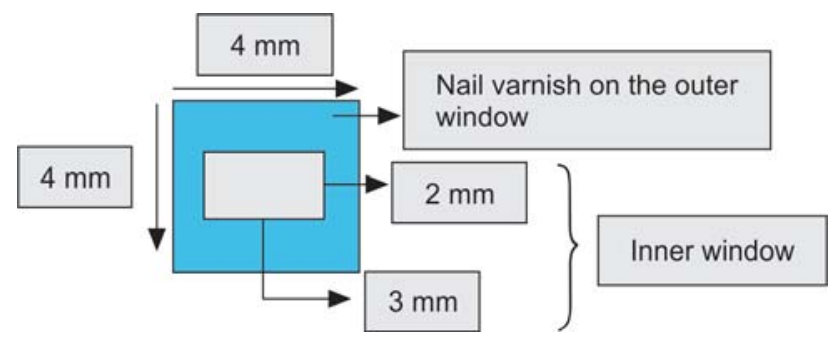

Fig. 1: Diagrammatic representation of the specimen used in the study

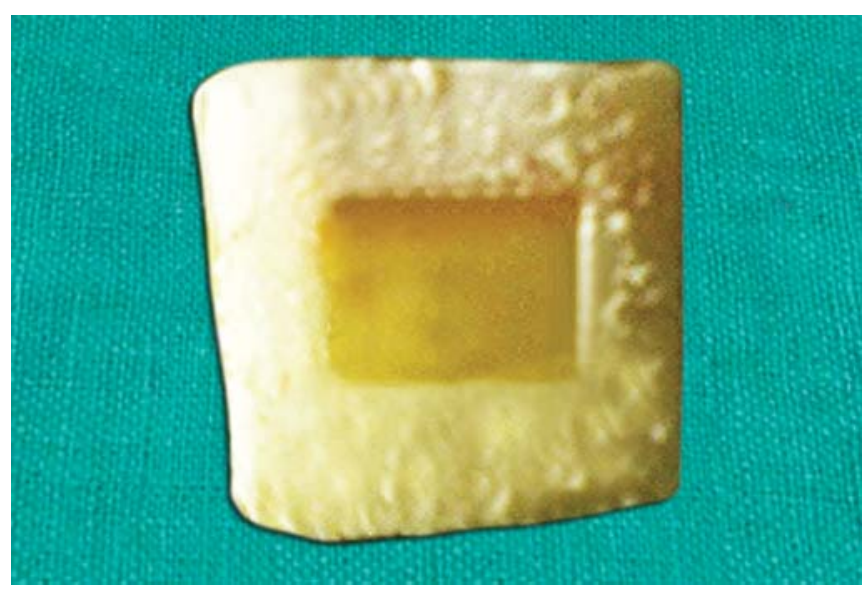

Fig. 2: The root fragment
A II the solutions were freshly prepared prior to use. All the samples were $\mathrm{pH}$ cycled in the following order. Treatment solutions (10 minutes), acidic buffer at pH 5.0 (30 minutes) and neutral buffer at pH 7.0 (10 minutes) for 8 days consisting of 6 cycles per day. Root fragments were stored in neutral buffer overnight (Fig. 3).

\section{Post-treatment Analysis and Confocal Laser Scanning Microscopy (CLSM)}

Following pH cycling, root fragments were rinsed with deionized water for 2 minutes. The fragments were stained with a freshly prepared $0.1 \%$ rhodamine B solution (A mitlal Chemicals Pvt Ltd, M umbai, India) for 1 hour and rinsed three times with deionized water for 5 minutes. The samples were analyzed with a confocal laser scanning microscopy (CLSM) (FV 1000 O LY M PUS), using a helium-neon laser with a $543 \mathrm{~nm}$ excitation wavelength. Theimages of stained posttreatment lesions were quantitatively analyzed for their optical densities with an image analysis system (FV V iewer). Interestingly, the optical density is inversely related to the porosity of the demineralized dentin, where increased porosity corresponds with decreased optical density. Hence, if remineralization occurs, the optical density will increase accordingly. ${ }^{9}$

The relative optical density (ROD) was calculated as follows:

- $O D_{R}=O D_{/} / O D_{S} \times 100 \%$

- Where $O D_{1}=O D$ of the lesion

- $O D_{S}=O D$ of sound root tissue.

\section{STATISTICAL ANALYSIS}

For each sample group, the mean and standard deviation were calculated. The data collected from the CLSM were analyzed using analysis of variance (ANOVA) with level of significance 0.05 .

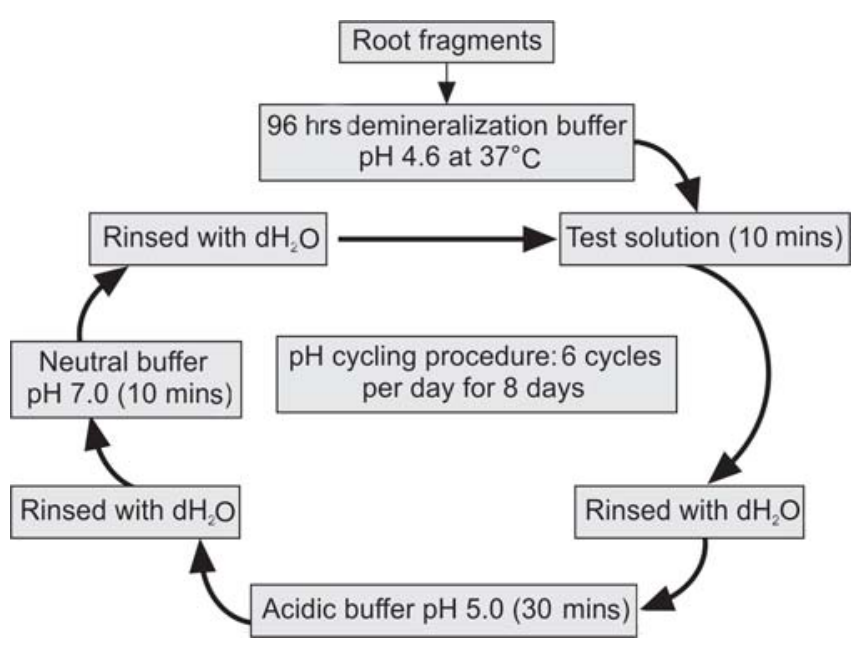

Fig. 3: Schematic representation of in vitro artificial lesion formation and $\mathrm{pH}$ cycling procedure 


\section{RESULTS}

There were significant differences between GSE and other treatments groups. When the stained samples were examined under the CLSM, a fluorescent red band was observed indicating the demineralized dentin (Figs $4 A$ to $D$ ). Lesions observed following demineralization after $\mathrm{pH}$ cycling showed an increase in fluorescence (less optical density) and those after treatment with remineralizing solutions showed a decrease in fluorescence (higher optical density). The ROD of GSE was significantly higher when compared with the other group which implies a higher degree of remineralization. The graph showing the distribution of ROD in each group is given in the figure below. From the
Table 1 and Figures 5 and 6 , it is evident that GSE showed best results among the materials compared.

\section{DISCUSSION}

Potent caries prevention activity has been attributed to hydrophilic fractions of natural extracts whose major components are high molecular weight substances like PA, consisting of approximately 22 catechin units in their structure ${ }^{7}$. GSE has high PA content. PA from GSE has been demonstrated to increase collagen synthesis and accelerate the conversion of soluble collagen to insoluble collagen during development. ${ }^{6}$ PA treated collagen matrices are nontoxic and inhibitenzymatic activity of glucosyltransferase,
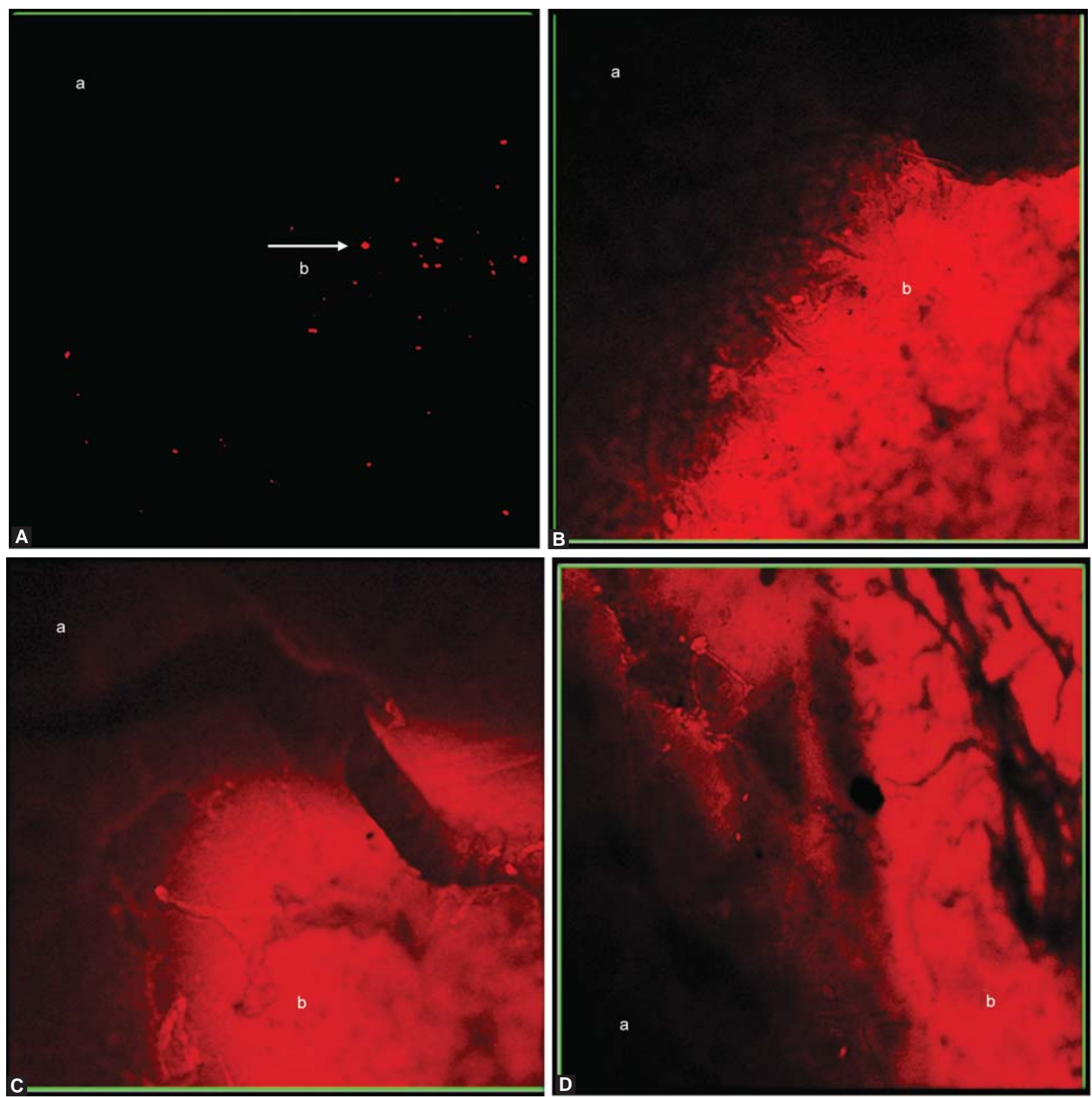

Figs 4A to D: Confocal laser scanning microscope images of artificial root lesions treated by: (A) $6.5 \%$ GSE, (B) $0.17 \%$ sodium monofluorophosphate and $0.05 \%$ CaGP, (C) $0.5 \% \mathrm{CaGP}$, (D) control (where $\mathrm{a}=$ sound dentin and $\mathrm{b}=$ fluorescence in the demineralized dentin) 
Table 1: ROD of artificial root lesions treated by GSE, $0.05 \%$ CaGP and $0.17 \%$ sodium monofluorophosphate, $0.5 \% \mathrm{CaGP}$ and deionized water

ROD of artificial root caries lesions for different treatment group*

\begin{tabular}{lcc}
\hline Groups & Mean & $\begin{array}{c}\text { Standard } \\
\text { deviation (SD) }\end{array}$ \\
\hline Group A (6.5\% GSE) & 78.37 & 12.43 \\
Group B (0.17\% sodium & 45.32 & 22.44 \\
monofluorophosphate with & & \\
$0.05 \%$ CaGP) & 41.52 & 20.74 \\
Group C (0.5\% CaGP) & 44.08 & 23.42 \\
Group D (control-no treatment) & 4 \\
\hline
\end{tabular}

*The greater the ROD, the greater the remineralization

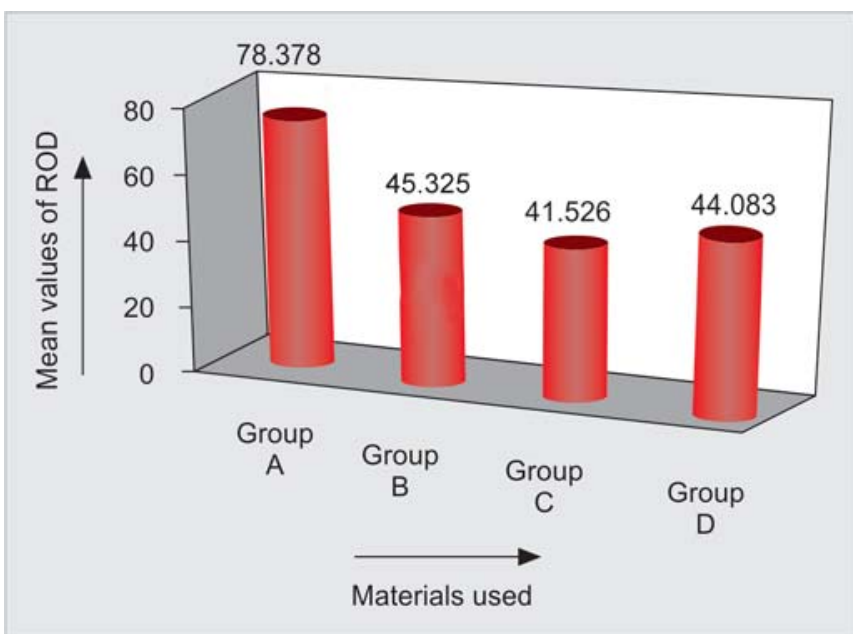

Fig. 5: The comparison of the different groups with respect to the mean value of ROD

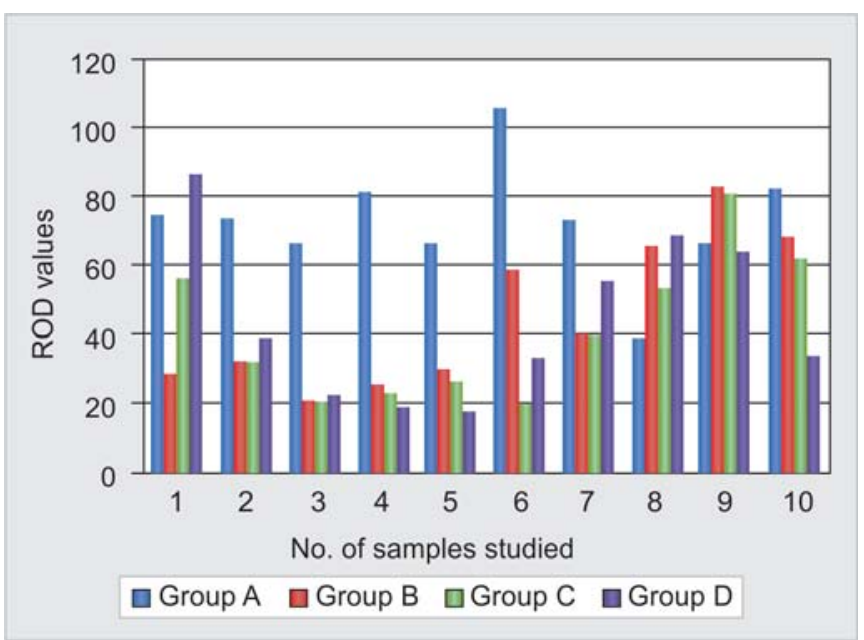

Fig. 6: Comparing the four groups with respect to their ROD values

F-ATPase and amylase. Glucosyltransferases which are produced by Streptococcus mutans polymerize the glucosyl moiety from sucrose and starch carbohydrates into glucans. This constitutes the sucrose-dependent pathway for
S.mutans to establish on tooth surface and is of central importance in plaque formation and development of caries. The adherent glucan also contributes to the formation of dental plaque, in which accumulation of acids leads to localized decalcification of enamel surface by facilitating bacterial adherence to tooth surfaces, inter bacterial adhesion and accumulation of biofilms. Hence, inhibition of glucosyltransferases by PA in turn inhibits caries., ${ }^{70,11}$ Bioflavanoids from other sources have al so been demonstrated to inhibit other oral Streptococci, Actinomycetes and Lactobacilli at concentrations of 1.56 to $6.25 \mathrm{mg} / \mathrm{mL} .{ }^{12}$

Grape seed extracts inhibit the growth of anaerobic bacteria, such as Porphyromonas gingivalis and F usobacterium nucleatum, associated with periodontal disease. ${ }^{11} \mathrm{As}$ we are investigating root caries, this may be a desirable and positive fall out of the anticariogenic effect.

The caries preventive effect of fluoride and calcium glycerophosphate has been proved beyond any doubt. A nticariogenic effect of fluoride can be attributed to various reasons including enhanced calcium/phosphate precipitation and supersaturation with respect to fluorapatite. ${ }^{13,14}$ Several possible reasons for anticaries potential of $\mathrm{CaGP}$ have been investigated. These include el evation of plaque calcium and phosphate concentrations, buffering plaque $\mathrm{pH}$, reduction of plaque mass and direct interaction with dental hard tissues as they act on the hydroxyapatite thereby reducing the extent of demineralization. Hence, fluoride and calcium glycerophosphate were used as positive controls. ${ }^{15-18}$

Grape seed extract was compared with fluoride and CaGP to assess its effect on remineralization process, using CLSM. CLSM is a nondestructive, three-dimensional technique of microscopic tomography. Confocal microscopy permits the study of unsectioned natural teeth. A rtefacts induced by sample preparation are thereby excluded. The intensity of the reflected laser light is visualized as pseudocolors. Light yellow to red colors stand for high intensities of reflected light from organic components. A reas of greater mineral content where the laser beam penetrates but is not scattered appear dark or black. ${ }^{19}$ Remineralized dentin shows greater optical density and appears darker than demineralized dentin. Interestingly, the relative optical density was higher in GSE than that of other groups in the present study. Hence, we can say that GSE positively affects the remineralization process. Proposed mechanism of its action is that it contributes to mineral deposition on the superficial layer of the lesion. GSE is said to form visually insoluble complexes when mixed with remineralizing solution at pH 7.4. GSE may interact with the organic portion of root dentin through PA - collagen interaction, thereby stabilizing the exposed collagen matrix. ${ }^{6}$ 
Grape Seed Extract as a Potential Remineralizing Agent: A Comparative in vitro Study

In vitro demineralization and remineralization studies are important research tools which provide vital information regarding clinical outcome. The dynamic and fluctuating variation in natural caries process is well-simulated by the in vitro $\mathrm{pH}$ cycling model used in this study. ${ }^{20,21}$

Root caries is highly prevalent in the elderly population, who would greatly benefit from noninvasive remineralization therapy. Hence, root caries lesions were targeted. ${ }^{22}$

The results of the present study corroborate and add to the results obtained in a previous study. ${ }^{6}$

Based on the resul ts obtained in this in vitro study, GSE inhibits demineralization and promotes remineralization of artificial root carious lesions better than fluoride and CaG P. The probable reasons for lesser activity of fluoride and CaGP compared to GSE include:

1. Sodium monofluorophosphate ( $220 \mathrm{ppm}$ ) used in the study needs to be broken down by salivary phosphatases in order to release fluorides and though the enzyme system is present in the saliva and plaque in vivo, it is absent in most in vitro methods. ${ }^{23}$

2. It has been established that the anticaries potential of CaGP may be greater when it is applied sometime before a cariogenic challenge than after or during a cariogenic challenge for effective remineralization. ${ }^{24}$

The study may be repeated with a larger sample size to validate the results and explain the large standard deviation values. Further studies are required to elaborate the mechanism of action and the clinical protocol of application of GSE.

\section{CLINICAL SIGNIFICANCE}

The search for the perfect remineralizing agent continues to this day. GSE could be a welcome addition to the remineralization armamentarium.

\section{ACKNOWLEDGMENTS}

W e wish to sincerely thank the National Centre for Biological Sciences, B engaluru, for permitting to use the confocal laser scanning microscope. M r M anoj M ullamangalam, statistician and $\mathrm{Dr}$ Naveen Sharma for help with statistical analysis. Thanks to our colleagues and family for their support.

\section{REFERENCES}

1. Mount GJ, Ngo H. M inimal intervention: A new concept for operative dentistry. Quintessence Int 2000;31:527-33.

2. Selvig KA. The fine structure of human cementum. A cta O dontol Scand 1965;23:423-41.

3. Furseth $R$, J ohansen $E$. The mineral phase of sound and carious human dental cementum studied by electron microscopy. A cta Odontol Scand 1970;28:305-22.
4. Furseth R. Further observations on the fine structure of orally exposed and carious human cementum. A rch Oral Biol 1971; 16:71-85

5. M ai S, K im Y K, K im J, Y iu CKY, Ling J, Pashley DH, et al. In vitro remineralisation of severely compromised bonded dentin. J Dent Res 2010;89:405-10.

6. Qian X ie, A na K arina B edran- R usso, Christine D W u. In vitro remineralisation effects of grape seed extract on artificial root caries. J Dent 2008;36:900-06.

7. Gianmaria F Ferrazzano, Ivana A mato, A niello Ingenito, A rmando Zarelli, Gabriele Pinto, A ntonino Pollio. Plant polyphenols and their anti-cariogenic properties: $A$ review. M olecules 2011;16:1486-507.

8. Christine D Wu. Grape products and oral health. Grapes and Health J N utr 2009; 139(9) (Suppl) S1818-23.

9. Gonzal ez-Cabezas C, Fontana M, Dunipace AJ, Li Y , Fischer $G M$, Proskin HM , et al. M easurement of enamel remineral isation using microradiography and confocal microscopy. A correlational study. Caries Res 1998;32:385-92.

10. Hattori $M, K$ usumoto I T, N amba T, Ishigami T, Hara Y . Effect of tea polyphenols on glucan synthesis by glucosyltransferases from Streptococcus mutans. Chem Pharm Bull 1990;38:717-20.

11. Sampaio FC, Pereira MS, Dias CS, Costa VC, Conde NC, Buzalaf M A I In vitro antimicrobial activity of Caesal pinia ferra $M$ artius fruits against oral pathogens. J Ethnopharmacol 2009; 15:289-94.

12. Timothe J, B onsi IA, Padilla-Zakour OI, Koo H. Chemical characterisation of red wine grape (V itis vinifera and $\mathrm{V}$ itis interspecific hybrids) and pomace phenolic extracts and their biological activity against Streptococcus mutans. J A gric Food Chem 2007;55,10200-207.

13. Ogaard B, Seppa L, Rolla G. Professional topical fluoride applications - clinical efficiency and mechanism of action. A dv Dent Res 1994;8:190-201.

14. Ten Cate JM . Remineralisation of deep enamel dentine caries lesions. A ust Dent J 2008;53:281-85.

15. B owen W H. The cariostatic effect of calcium glycerophophate in monkeys. Caries Res 1972;6:43-51.

16. Duke SA, Rees DA, Forward GC. Increased plaque calcium and phosphorous concentrations after using a calcium carbonate toothpaste containing calcium glycerophosphate and sodium monofluorophosphate. Caries Res 1979;13:57-59.

17. Nordbo H, Rolla G. Desorption of salivary proteins from hydroxyapatite by phytic acid and glycerophosphate and the plaque inhibiting effect of the two compounds in vivo. J Dent Res 1972;51:800-11.

18. Grenby TH, Bull JM. U se of high performance liquid chromatography techniques to study the protection of hydroxyapatite by fluoride and glycerophosphate against demineralization in vitro. Caries Res 1980;14:221-32.

19. Bjorn Ogaard, Heinz Duschner, Jan Ruben, J oop A rends. $M$ icroradiography and confocal laser scanning microscopy applied to enamel lesions formed in vivo with and without fluoride varnish treatment. Eur J oral Sci 1996;104:378-83.

20. White DJ. The application of in vitro models to research on demineralisation and remineralisation of the teeth. Adv Dent Res 1995; 9:175-93.

21. Exterkate RA M , Damen JJM , Ten Cate J M. Single section model for enamel de- and remineralisation studies. I. The effects of 
different $\mathrm{C}$ a/P ratios in remineralisation solutions. J Dent Res 1993; 72:1599-603.

22. David B Scott, James W Simmelink, V ibeke Nygaard. Structural aspects of dental caries. J Dent Res 1974;53:165-78.

23. Marilia A fonso Rabelo Buzalaf, Angelica Reis Hannas, Ana Carolina M ahalhaes, Daniela Rios, Heitor M arques Honorio, A lberto Carlos Botazzo Delbem. PH cycling models for in vitro evaluation of the efficiency of fluoridated dentrifices for caries control: strengths and limitations. J A ppl Oral Sci 2010;18:316-34.

24. Lynch RJ M, T en Cate J M. Effect of cal cium glycerophosphate on demineralisation in an in vitro biofilm model. Caries Res 2006;40:142-47.

\section{ABOUT THE AUTHORS}

\section{Shiny Benjamin}

Reader, D epartment of C onservative D entistry and Endodontics, V ydehi Institute of D ental Sciences, B engaluru, K arnataka, India

\section{Roshni}

Senior L ecturer, Department of Conservative Dentistry and Endodontics, Vydehi Institute of Dental Sciences, Bengaluru Karnataka, India

\section{Sabeena Susan Thomas (Corresponding Author)}

Postgraduate Student, Department of Conservative Dentistry and Endodontics, V ydehi Institute of D ental Sciences, N 0. 82 E PIP A rea Nallurahalli, W hitefield, B engaluru-560066, K arnataka, India Phone: 00919739869119, 080-28413381, Fax: 080-28416199 e-mail: sabeena.susans.thomas@gmail.com

\section{Mohan Thomas Nainan}

Professor and Head, Department of Conservative Dentistry and Endodontics, V ydehi Institute of Dental Sciences, B engaluru Karnataka, India 